\title{
El Tratado de Álgebra elemental de Juan Cortázar: un libro significativo para la enseñanza de las matemáticas en España
}

\section{Juan Cortázar's Elementary Treatise on Algebra: A relevant book for teaching mathematics in Spain}

\author{
Carmen León-Mantero ${ }^{1}$ \\ cmleon@uco.es \\ Universidad de Córdoba, España \\ Alexander Maz Machado \\ ma1mamaa@uco.es \\ Universidad de Córdoba, España \\ María José Madrid Martín \\ mjmadridma@upsa.es \\ Universidad Pontificia de Salamanca, España
}

\section{Resumen:}

En las últimas décadas, ha ido creciendo el interés de los investigadores en Historia de las Matemáticas y Educación Matemática, por analizar los libros de texto de Matemáticas que han tenido influencia en su enseñanza, bien por la relevancia de su autor o por su amplio uso o difusión. En este trabajo se presenta el análisis del libro de texto español Tratado de Álgebra Elemental del autor del siglo XIX Juan Cortázar, uno de los primeros Catedráticos de matemáticas, cuyos libros de texto fueron elegidos para formar parte de las listas oficiales para la enseñanza en secundaria desde el año 1848 y siguieron reimprimiéndose hasta entrado el siglo XX. Se plantea un estudio descriptivo y cualitativo mediante la técnica del análisis de contenido. Se hallaron diversas estrategias

\begin{abstract}
:
Over the last decades researchers in the History of Mathematics and Mathematics Education have developed a growing interest in analyzing mathematics textbooks which have influenced the teaching of this subject. This increase has been mediated by the relevance of the authors of the manuals or by the widespread use of those manuals. This study presents the analysis of the Spanish textbook Tratado de Algebra Elemental (Elementary treatise on algebra) written by the 19th century author, Juan Cortázar, one of the first mathematics professors in Spain, whose books were approved for secondary education in 1848 and continued being reprinted until the 20th century. We designed a descriptive and qualitative study using the content analysis technique. The results show that the book included several didactic strate-
\end{abstract}

1 Dirección para correspondencia (correspondence address):

Carmen León-Mantero. Facultad de Ciencias de la Educación, San Alberto Magno s/n, 14004 Córdoba (España). 
El Tratado de Álgebra elemental de Juan Cortázar: un libro significativo para la enseñanza de las matemáticas en España

Carmen LeÓn-Mantero, Alexander Maz-Machado y María José Madrid Martín

didácticas, y se categorizaron los sistemas de representación y los aspectos fenomenológicos que el autor utilizó para modelizar numerosas situaciones mediante los conceptos matemáticos que se expone en la obra.

\section{Palabras clave:}

Historia de la Educación Matemática; Juan Cortázar; siglo XIX; análisis de contenido. gies. Representation systems and the phenomena which Cortázar used to model numerous situations through the mathematical contents included in the books were also categorized.

\section{Keywords:}

History of Mathematics Education; Juan Cortázar; nineteenth century; content analysis.

\section{Résumé:}

Au cours des dernières décennies, il y a eu un intérêt croissant de chercheurs en histoire des mathématiques et de l'enseignement des mathématiques, d'analyser les manuels de mathématiques qui ont influencé son enseignement, soit par la pertinence de son auteur ou de son utilisation généralisée ou par diffusion. Dans cet article, I'analyse du Manuel Espagnol Tratado de Álgebra Elemental (Traité de ElementaryAlgebra) de l'auteur du dix-neuvième siècle, Juan Cortazar, I'un des premiers professeurs de mathématiques est présenté, dont les manuels ont été choisis pour faire partie des listes officielles pour l'enseignement de l'année secondaire. Ils sont réimprimés en 1848, et ont continué d'être imprimés au cours du vingtième siècle. Une étude descriptive et qualitative a été proposée par la technique de l'analyse de contenu. Diverses stratégies d'enseignement ont été faites, et les systèmes de représentation et les aspects phénoménologiques que l'auteur utilise pour modéliser de nombreuses situations en utilisant des concepts mathématiques seront énoncés dans ce travail.

\section{Mots-clés:}

Histoire de l'enseignement des mathématiques, Juan Cortázar, XIXème siècle, I'analyse du contenu.

Fecha de recepción: 09-07-2016

Fecha de aceptación: 03-01-2018

\section{Introducción}

La investigación histórica en Educación Matemática centrada en los libros de texto proporciona información sobre el estado del conocimiento científico de distintas épocas y puede ayudar a comprender los avances de los planes de estudio vigentes en el sistema educativo de cada momento (Maz y Rico, 2015).

Es por ello que, en los últimos años se han realizado numerosas investigaciones sobre la Historia de las Matemáticas y de la Educación Matemática y, en particular, sobre el análisis de manuales de texto. A nivel internacional, destacan trabajos que defienden que la Historia de las Matemáticas es un elemento fundamental a tener en cuenta a la hora de enseñar matemáticas (Fauvel, 1991; Furinghetti, 2000; Jankvist, 2009; 
Tzanakis y Arcavi, 2000; Wussing, 1998). También se han realizado trabajos específicos sobre libros de texto antiguos. Así por ejemplo Frejd (2013) analiza y compara libros antiguos de álgebra publicados en Suecia, entre 1794 y 1836. Schubring (1988) analiza manuales de matemáticas alemanes y franceses para determinar el tratamiento que se le daba a los números negativos en la primera mitad el siglo XIX. El propio Schubring (1987) propone algunos aspectos metodológicos para su análisis.

En España también hay una corriente de investigación histórica sobre libros antiguos de matemáticas. En esta línea Gómez (2011a; 2011b) establece un marco para su estudio a nivel español y señala cómo identificar problemas de investigación en esta temática. Destacan los trabajos españoles de Sierra, González y López (1999; 2003) sobre los conceptos de límite y continuidad en los libros de texto españoles, Maz (2005) y Maz y Rico (2007; 2009a) sobre el estudio de los números negativos en los manuales españoles de los siglos XVIII y XIX. También los de Maz y Rico (2009b; 2013) sobre el estudio de personajes históricos como autores de libros de texto.

La evolución histórica de los conceptos y ámbitos matemáticos constituye uno de los cinco organizadores curriculares, es decir, es una de las componentes fundamentales para articular el diseño, desarrollo y evaluación de unidades didácticas, cuya finalidad es la de "señalar algunos momentos a lo largo de la historia de la matemática en los que el conocimiento matemático considerado tuvo un desarrollo especial o desarrolló algún papel de interés" (Rico, 1997, p.55).

Debido al carácter educativo que poseen, en los libros de texto se encuentran dos de los organizadores curriculares propuestos por Rico (1997):

- Los fenómenos o situaciones en las que se presentan y emplean los conocimientos y las aplicaciones prácticas de cada bloque de contenidos, y

- Las representaciones utilizadas en cada concepto, es decir, el modo simbólico o gráfico por el cual se expresan los conocimientos matemáticos.

Este trabajo centra su atención en estos dos organizadores. El objetivo de este trabajo es analizar el libro de texto español titulado Tratado de Álgebra Elemental de Juan Cortázar, mediante un análisis de contenido centrado en los sistemas de representación, sistemas fenomenológicos y 
El Tratado de Álgebra elemental de Juan Cortázar: un libro significativo para la enseñanza de las matemáticas en España

Carmen León-Mantero, Alexander Maz-Machado y María José Madrid Martín

contenido matemático hallado. La relevancia de este tratado se manifiesta en diversos aspectos. Fue publicado por primera vez en 1848 y, reeditado en cuarenta ocasiones, estando las últimas ediciones publicadas en la primera parte del siglo XX. Por otro lado, su autor, Juan Cortázar, fue uno de los dos primeros Catedráticos de Matemáticas en España, en las cátedras de Matemáticas Elementales del Instituto de Noviciado de Madrid y de Álgebra superior y Geometría Analítica en la Facultad de Ciencias de la Universidad Central de Madrid. Sus libros fueron seleccionados para formar parte del listado oficial de libros de texto para la enseñanza secundaria en 1848 y siguieron figurando en los posteriores listados oficiales que se actualizaban cada curso escolar (Vea, 1995).

\section{Metodología}

La metodología usada en este estudio es el análisis de tipo histórico, en particular el análisis de contenido. Éste se realiza alrededor de tres focos, pautados en el trabajo de Gómez (2002): la estructura conceptual, los sistemas de representación y la fenomenología.

La estructura conceptual identifica los conceptos, y las relaciones entre ellos, del contenido matemático. Los sistemas de representación son las diferentes formas de expresar un concepto o una estructura matemática. El análisis fenomenológico identifica las situaciones en las que los conceptos matemáticos se plantean.

Este trabajo se basa en los estudios realizados en investigaciones anteriores de similares características dentro del grupo de Historia de las matemáticas y la Educación matemática de la Sociedad Española de Investigación en Educación Matemática (SEIEM) en las que se usa como método el análisis de contenido aplicado a los libros de texto de matemáticas (Maz y Rico, 2009a; Madrid, Maz-Machado y León-Mantero, 2015).

Se fijaron como categorías de análisis, los conceptos, las imágenes, representaciones, los ejemplos y las actividades propuestas.

En primer lugar, se analizó la secuenciación de los contenidos, ejemplos, ejercicios y problemas que se encuentran en la obra y la interrelación entre ellos. Además, se construyó un mapa conceptual que muestra los focos conceptuales en los que se estructura la obra.

Por otro lado, se clasificaron los sistemas de representación hallados, entendiendo por representaciones, las formas de expresar de manera 
simbólica o gráfica los conceptos matemáticos y sus propiedades más importantes, como por ejemplo son las representaciones verbales, algebraicas, numéricas o gráficas (Castro y Castro, 1997).

Por último, se examinaron los contextos o situaciones sociales, naturales y matemáticas, que se encuentran presentes en los conceptos, ejemplos y ejercicios propuestos en la obra. El análisis fenomenológico muestra cómo se usan y se aplican los conceptos y la manera de abordar los distintos ejemplos y ejercicios (Rico, Lupiáñez, Marín y Gómez, 2008).

\section{Resultados}

El apartado resultados se ha dividido en cinco secciones. La primera consiste en una breve semblanza del autor, que recorre la vida académica y profesional de Juan Cortázar, y sirve de contextualización histórica, social y cultural de la época. A continuación, se describe la caracterización de la obra, en el que se incluye el número de ediciones del libro de texto, el público al que va dirigido, la extensión, estructura y secuenciación de los contenidos, así como la información que aporta la portada y el prólogo de la edición. Las tres últimas secciones corresponden al análisis de los resultados obtenidos sobre los aspectos que ya han sido desarrollados en el apartado metodológico: la estructura conceptual, los sistemas de representación y el análisis fenomenológico de la obra. Los ejemplos que ilustran los resultados, se han reproducido textualmente, conservando la grafía original y sin adaptar ni la acentuación ni la puntuación a la actual.

\section{a. El autor: Juan Cortázar Abasolo² (1809-1873)}

Nació en Bilbao el 8 de junio ${ }^{3}$ de 1809, teniendo en cuenta la baja tasa de escolarización de la época, podemos considerar a Cortázar un individuo afortunado por tener la oportunidad de estudiar latín en el convento de los Franciscanos de Bilbao, desde los 10 hasta los 13 años, y después realizar los estudios de Humanidades y los idiomas de francés e inglés

2 El apellido Abasolo aparece escrito también Albásolo según la literatura consultada. Hemos decidido usar Abasolo por aparecer un mayor número de veces.

3 El mes de nacimiento aparece en muchos escritos como el de julio. Análogamente nos inclinamos a escribir junio por aparecer en un número mayor de ocasiones 
El Tratado de Álgebra elemental de Juan Cortázar: un libro significativo para la enseñanza de las matemáticas en España

Carmen León-Mantero, Alexander Maz-Machado y María José Madrid Martín

en el Colegio de Santiago hasta tener cumplidos los 18 años de edad. Trabajó como profesor de Matemáticas en el mismo colegio en el que estudió hasta el año 1834 en el que fue becado para estudiar en la Escuela Central de Artes y Manufacturas de París (Irueste, 1912).

Escribió un tratado completo de trigonometría en 1838, mientras vivía aún en París, que nunca llegó a publicarse, pero gracias al cual dedujo una demostración elegante y original de las analogías de Neper, que fue publicada en el periódico francés titulado Nouvelles annales de mathématiques en 1847 (Cortázar, 1847).

Al regresar a España, ya con su título de Ingeniero fue nombrado Catedrático de Matemáticas Elementales del Instituto de Noviciado de Madrid, para dar respuesta a la incorporación de estudios de segunda enseñanza en la Facultad de Filosofía y así dividir estos estudios en elementales y superiores de Filosofía. Supo compatibilizar su docencia con la dirección del Colegio Politécnico, del Colegio Marsanau y de una Academia de Matemáticas a la que bautizó con su nombre, dando respuesta a las necesidades de los continuos cambios de planes de estudio que se produjeron desde 1837 (Rodríguez, 2009).

Se licencia en Ciencias Físico-Matemáticas en 1847, y, en 1850 es nombrado Catedrático de Álgebra superior y Geometría analítica de la Facultad de Filosofía, en su sección de Ciencias de la Universidad Central, en la que más tarde ocupará el título de decano (Rodríguez, 2009). Como afirman Peset et al. (1978), Gil de Zárate realizó un escalafón con el número de orden, nombre, Universidad, facultad y asignaturas que impartían los catedráticos de la Universidad española. Cortázar aparece como uno de los dos catedráticos de matemáticas, junto a Francisco Travesedo, en 1851 y, tras la jubilación de éste, en el nuevo escalafón del 1859 aparece solo Cortázar.

Cortázar fue docente de Álgebra superior y Geometría analítica en la Facultad de Filosofía hasta el año 1857 en el que la Ley Moyano separó los estudios de ciencias de ésta y organizó la Universidad de Ciencias en tres secciones: físico-matemáticas, químicas y naturales. En las dos primeras impartía Geometría analítica, y en la sección de naturales, la asignatura de Álgebra (Universidad de Madrid, 1856; 1857).

Ese mismo año fue elegido miembro de la Real Academia de Ciencias Exactas, aunque debido a problemas de salud no llegó a ser miembro activo ni a leer su discurso de ingreso, sin embargo, continuó ejerciendo como profesor hasta su fallecimiento (Irueste, 1912). 
Cortázar muere en Madrid el 12 de abril de 1873 a los 63 años de edad a consecuencia de un tumor. Gumersindo Vicuña, en su discurso del acto de apertura del curso académico 1875-1876, en el que dedica unas palabras a profesores recientemente fallecidos, como Valledor, Bengoechea, Presas, Elizalde, Rey Heredia y, por supuesto Cortázar, describe a nuestro autor como "el matemático Cortázar, carácter de oro con corteza de barro, espíritu original, autor metódico y claro" (Vicuña, 1875, p. 31).

Varios alumnos suyos se convirtieron en ilustres profesores de matemáticas, como Hipólito Díaz Pardo y Botaz, y José Andrés Irueste, quien le sustituye al frente de sus clases al caer Cortázar enfermo. Este utilizó sus obras como libros de texto hasta el año 1876 y dedicó a su maestro la publicación de su libro en una emotiva nota en el que se refiere a él como "eminente matemático, insigne profesor y probo ciudadano" (Girón y Girón, 2010, p. 355). En el XXXIX aniversario de la muerte de su maestro, a Irueste le fue encomendada la tarea de escribir una semblanza de su vida y un análisis de su obra en la Revista de la Sociedad Matemática Española. En ella, comparó intelectual y moralmente a Cortázar con el destacado filósofo y matemático cordobés D. José Rey y Heredia (Irueste, 1912).

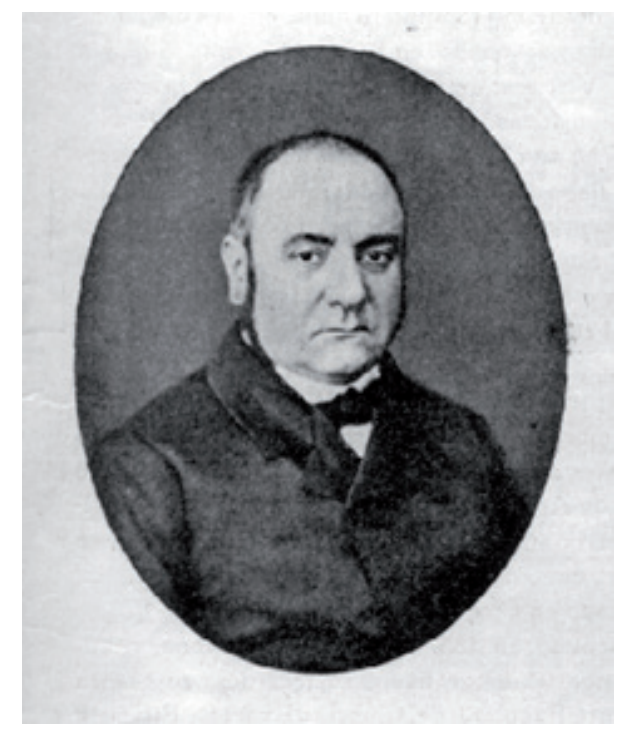

Figura 1. Juan Cortázar Abásolo (Irueste, 1912) 
El Tratado de Álgebra elemental de Juan Cortázar: un libro significativo para la enseñanza de las matemáticas en España

Carmen LeÓn-Mantero, Alexander Maz-Machado y María José Madrid Martín

\section{b. La obra: Tratado de Álgebra Elemental}

Para el análisis del Tratado de Álgebra elemental, se eligió la decimoquinta edición, impresa en Madrid en el año 1865 en la Imprenta de Antonio Peñuelas y Gabriel Pedraza, ejemplar que forma parte de la biblioteca de la Universidad Complutense de Madrid.

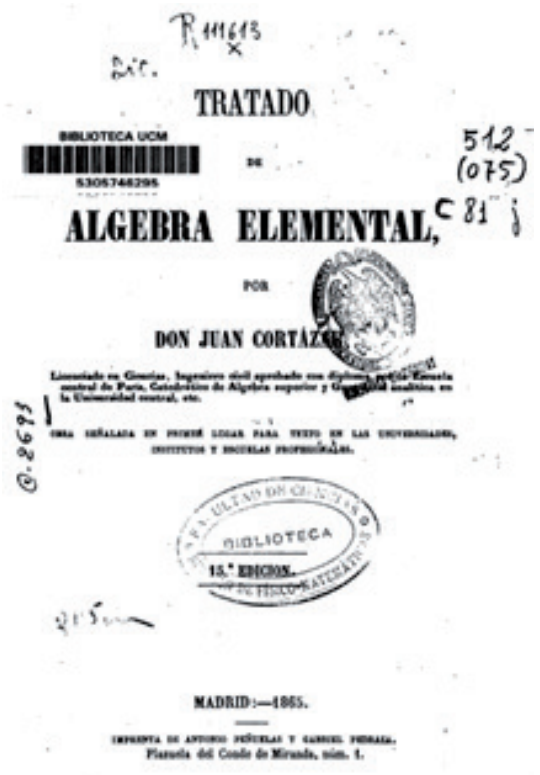

Figura 2. Portada del Tratado de Álgebra Elemental por Juan Cortázar, 1865

El tratado se editó por primera vez en 1848 y, tras 40 reediciones, en 1919, su hijo Daniel Cortázar y Larrubia, publicó la última de ellas, 46 años después de la muerte de su padre. Está formado por un solo tomo de 216 páginas, organizado en 6 libros, éstos a su vez divididos en 33 capítulos. Los 6 libros están dedicados respectivamente al cálculo algébrico, las ecuaciones de primer grado, los problemas determinados de primer grado, las potencias y raíces de las cantidades algebraicas, las ecuaciones de segundo grado y los logaritmos y progresiones.

La obra usa un lenguaje formal, está constituida por definiciones, teoremas con sus respectivas demostraciones, corolarios, métodos de resolución algebraicos y ejemplos variados. En ella, se intercalan diferentes capítulos dedicados a resolver problemas mediante ecuaciones de primer y segundo grado. 
Tabla 1

Índice del Tratado de Álgebra Elemental de Juan Cortázar (1865)

\begin{tabular}{ll}
\hline LIBRO $1^{\circ}$ - Cálculo algébrico & Págs. 4-41 \\
LIBRO $2^{\circ}$ - Ecuaciones de primer grado & Págs. 42-72 \\
LIBRO $3^{\circ}$ - Problemas determinados de primer grado & Págs. 73-94 \\
LIBRO $4^{\circ}$ - Potencias y raíces de las cantidades algébricas & Págs. 95- 134 \\
LIBRO $5^{\circ}$ - Ecuaciones de segundo grado & Págs. 135-166 \\
LIBRO $6^{\circ}$ - Logaritmos y progresiones & Págs. 167-216 \\
\hline
\end{tabular}

Se encuentran pocas referencias a otros autores en el texto. Entre ellas, cita a Descartes, para establecer su principio sobre las ecuaciones que se plantean para resolver problemas y a Neper, Briggs, Lalande y Marie como inventores y constructores de las tablas de logaritmos. Sin embargo, hallamos referencias continuas a su Tratado de Aritmética y Complemento de la Aritmética siempre que el autor necesita ampliar información o usar un concepto o resultado previo.

\section{c. Sobre la estructura conceptual}

Utilizando metodología inductiva, el tratado comienza con el planteamiento de un problema que resuelve aritméticamente, para después deducir un resultado general. De esta forma, el autor concluye: "Acabamos de ver que por medio del lenguaje simbólico o razonamiento artificial se simplifica mucho la resolución de los problemas. Este lenguaje simbólico o razonamiento artificial, aplicado a la resolución de los problemas matemáticos, se llama álgebra" (p. 5). Tras dar las diversas definiciones que forman el cálculo de expresiones algebraicas, el libro desarrolla las operaciones de monomios, polinomios y fracciones algebraicas, aunque en la obra se usa el término algébrico, en vez de algebraico, tal y como se usa en la actualidad.

Cabe destacar la importancia que le da a que los alumnos sepan distinguir entre identidad y ecuación dedicándole un apartado especial para ello. Así mismo, resalta la conveniencia de considerar la incógnita de una ecuación como representante tanto de un número positivo como uno negativo, para así poder generalizar la misma ecuación para dar soluciones en ambos sentidos. Es por ello que dedica la mayor parte del capítulo II del libro primero a las operaciones y propiedades de los números negativos. Además, se vale del uso de los paréntesis y las pro- 
El Tratado de Álgebra elemental de Juan Cortázar: un libro significativo para la enseñanza de las matemáticas en España

Carmen León-Mantero, Alexander Maz-Machado y María José Madrid Martín

piedades asociativa y conmutativa para expresar todos los casos posibles a los que se puede enfrentar el alumno a la hora de calcular y simplificar expresiones algebraicas.

El último artículo del libro primero Interpretación de las expresiones a/0 y $0 / 0$, incluye tres proposiciones de las cuales se obtienen cinco resultados. Para deducir el resultado de la expresión a/0, Cortázar estudia la tendencia de la fracción $a / b$ siendo $b$ un valor de la forma $1 / 10^{n}$, al que nombra "cantidad infinitamente grande, ó de infinito" (p. 39). Como consecuencia, se puede demostrar que a/ø tendrá un valor de cero.

El segundo resultado estudia el valor de la fracción $0 / 0$, demostrando primero que es un valor indeterminado por ser la solución de la ecuación $a \cdot 0=0$. Desde este punto y haciendo uso de cuatro ejemplos, elegidos específicamente para ello, demuestra cómo calcular el valor numérico de fracciones algebraicas cuando el resultado queda en términos de $0 / 0$.

De la misma manera, y tras dar las definiciones y propiedades correspondientes al cálculo de ecuaciones de primer grado, en el libro segundo establece cuatro pasos para resolver las ecuaciones con una incógnita: "quitando denominadores, efectuando las operaciones indicadas, haciendo la transposición y la reducción, es claro que la ecuación de primer grado con una incógnita tendrá la forma $a x=b$ de donde resulta $X=b / a^{\prime \prime}$ (p.49). Además dedica un pequeño apartado a resolver ecuaciones de primer grado con una incógnita en el que la incógnita aparece en el denominador y en las que aparecen radicales, explicando que en los casos en los que se multipliquen o dividan ambos miembros de la ecuación por cantidades desconocidas, así como elevando a una potencia o extrayendo raíces, se debe comprobar que las soluciones dadas son realmente soluciones de la ecuación ya que el número de soluciones puede variar.

Tras ofrecer numerosos ejemplos de cada uno de los pasos que deben seguirse para resolver una ecuación de primer grado, propone algunos ejemplos con su solución correspondiente, pero sin el procedimiento resuelto, para que los alumnos los resuelvan instándoles a comprobar la solución obtenida al finalizar el ejercicio y así evitar que se hayan cometido errores.

Para finalizar, enuncia un resultado que dice que una ecuación de primer grado con una sola incógnita solo tendrá una solución, debido a que todas pueden reducirse a la forma $a \cdot X=b$, cuya única solución es $x=b / a$. 
En el caso de los métodos de resolución de dos o más ecuaciones con dos o más incógnitas, Cortázar desarrolla los métodos de sustitución, de adición o sustracción y de igualación para demostrar que cualquiera de estos métodos de eliminación nos proporciona ecuaciones equivalentes a las dadas. Por ese motivo, en el caso en el que queramos resolver un sistema de ecuaciones de primer grado con igual número de incógnitas, "se elimina una incógnita entre dichas ecuaciones, y resultará una ecuación menos y una incógnita menos. Se vuelve a eliminar una incógnita en estas ecuaciones; y continuando del mismo modo, se llegará a una ecuación con una incógnita" (p. 58). Tras resolver el valor de dicha incógnita, el cálculo de las demás será fácil.

Al igual que en el caso de las ecuaciones de primer grado, recomienda la comprobación de los valores obtenidos y propone varios ejemplos con su solución correspondiente que sirva de apoyo al alumno.

El libro tercero está dedicado a problemas que pueden resolverse mediante ecuaciones de primer grado. El autor plantea todos los problemas y los resuelve paso a paso siguiendo el siguiente esquema: definición de los datos, planteamiento, resolución y comprobación. La mayoría de los ejemplos de este capítulo hacen referencia a situaciones de la vida cotidiana y, como él mismo apunta, son susceptibles de ser resueltos mediante operaciones aritméticas sin necesidad de usar lenguaje algebraico, sin embargo, "por medio del lenguaje simbólico ó razonamiento artificial se simplifica mucho la resolución de los problemas" (p.5).

Cortázar aclara que la mayoría de los problemas que resuelve a través de ecuaciones de primer grado con una sola incógnita, también pueden resolverse mediante varias incógnitas, y, por tanto, realiza de nuevo los problemas, representando cada una de las incógnitas con una letra diferente. Por último, resuelve de nuevo los ejemplos usando datos generales en lugar de datos particulares.

El libro cuarto, titulado Potencias y raíces de las cantidades algébricas, se centra principalmente en las propiedades del producto, cociente y potencia de potencias y raíces de monomios y de polinomios. Cortázar acepta la definición de raíz de un número como el "número que elevado a la potencia cuyo exponentes el índice de la raíz produce el número propuesto" (p. 97) con la consecuencia de que "Todo número positivo tiene dos raíces de grado par, las cuales solo se diferencian en el signo: pues un número positivo o negativo elevado a la potencia de grado par da un resultado positivo" (p. 97). Por tanto, el autor debe adoptar un con- 
El Tratado de Álgebra elemental de Juan Cortázar: un libro significativo para la enseñanza de las matemáticas en España

Carmen León-Mantero, Alexander Maz-Machado y María José Madrid Martín

venio que le evite confusiones y llama raíz aritmética o valor aritmético de la raíz de un número al valor de la raíz real positiva de éste.

Para calcular las potencias de los polinomios, define permutaciones y combinaciones de grupos de elementos y deduce con antelación la fórmula del binomio de Newton para expresiones del tipo $(a+b)^{n}$.

El último capítulo del libro cuarto, titulado Cálculo de las cantidades imaginarias de segundo grado, está destinado al cálculo de las raíces cuadradas de cantidades negativas. Tras definirlas como cantidades de la forma $a \pm b \sqrt{ }-1$ siendo a su término real y $b$ su parte imaginaria, detalla las operaciones de suma, diferencia, producto, cociente, potencias sucesivas y raíces cuadradas.

En el libro quinto, desarrolla la resolución de ecuaciones de segundo grado, clasificándolas en incompletas, completas, bicuadradas y resolución de dos ecuaciones con dos incógnitas. Para la ecuación $a X^{2}+b X+c=0$ demuestra las soluciones $X=-m / 2 \pm \sqrt{ }\left(m^{2} / 4-n\right)$ siendo $m=b / a$ y $\mathrm{n}=\mathrm{c} / \mathrm{a}$, las soluciones $X=\left(-b \pm \sqrt{ }\left(b^{2}-4 a c\right)\right) / 2 a$ y $x=r$ y $X=s$, siendo $r+s=-b / a$ y $r s=c / a$.

Dedica un apartado a la discusión del número y tipo de soluciones en función del discriminante y, por tanto, de los coeficientes de la ecuación. $\mathrm{Al}$ igual que el libro tercero, el quinto contiene problemas que pueden resolverse mediante ecuaciones de segundo grado con la solución corespondiente.

A continuación, aunque el autor especifica que el cálculo de máximos y mínimos es una cuestión del Cálculo Diferencial, lo explica para el caso de funciones de segundo grado. Para ello, se despeja la función y se resuelve y se discute los valores máximos y mínimos que se pueden obtener.

Los logaritmos y progresiones son estudiados en el libro sexto. Cortázar introduce la teoría sobre logaritmos estudiando las tendencias al infinito y las propiedades de las funciones exponenciales. De ese modo, define:"el logaritmo de un número es el exponente a que debe elevarse una cantidad positiva y diferente de 1, llamada base, para que la potencia sea igual al número" (p. 172). Incluye las propiedades y operaciones de los logaritmos, así como el método de construcción y el uso de la tabla de logaritmos de base 10. Como complemento, incluye la resolución de ecuaciones exponenciales, a través de una serie de ejemplos y un problema, en los que estudia la equivalencia entre logaritmos de distinta base. 
En el siguiente capítulo, se estudian los términos generales y la suma de las progresiones aritméticas y geométricas, así como la interpolación de diferentes elementos, entre varios números. En este caso, en contraposición con los capítulos anteriores, el autor utiliza la resolución de problemas en vez de ejercicios para aportar los ejemplos necesarios. Los problemas versan sobre cálculo de distancias y tiempos. Plantea, asimismo, el problema que habla sobre el inventor del ajedrez que se puede encontrar de forma similar en libros españoles dedicados a la enseñanza de las matemáticas escritos en el siglo XVI, como por ejemplo en la obra de Juan de Ortega. También se incluyen las aplicaciones que estudian el número total de balas que podían colocarse en las pilas de balas que se formaban en los parques de artillería o las que estudian los intereses generados por las anualidades o rentas vitalicias.

En la Figura 3 se presenta el mapa conceptual de la obra.

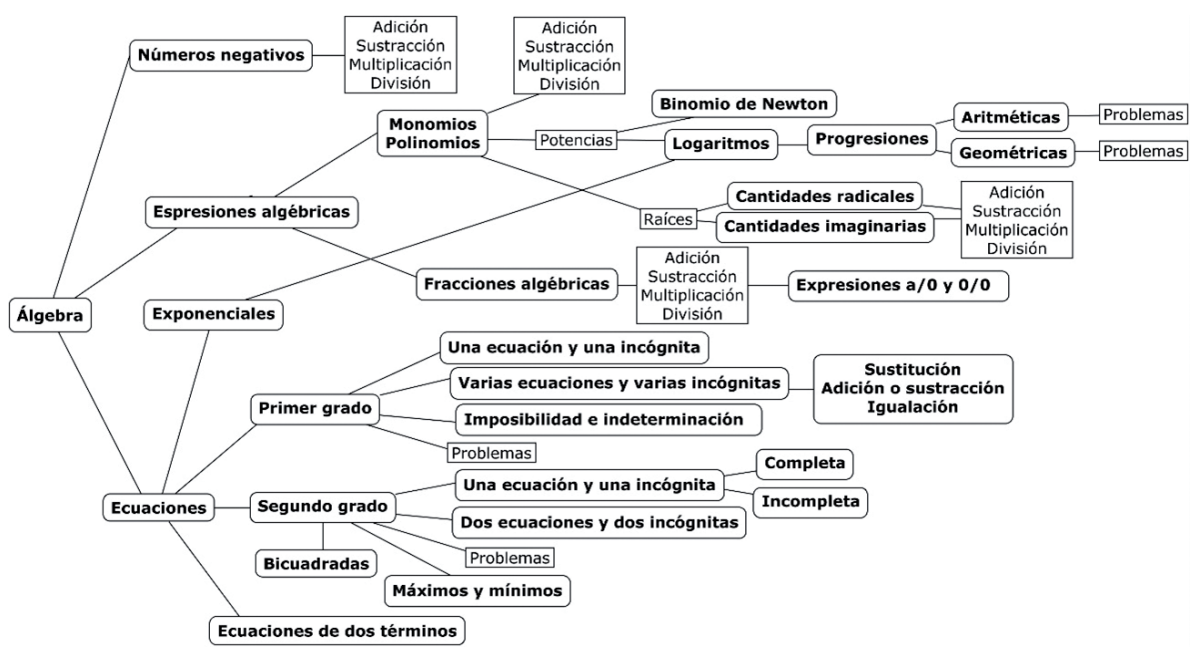

Figura 3. Mapa conceptual del Tratado de Álgebra Elemental de Juan Cortázar, 1865.

\section{d. Los sistemas de representación}

Se han localizado cuatro tipos de representaciones en el texto: algebraicas, numéricas, gráficas, y lenguaje verbal. Esta clasificación está basada y adaptada de la propuesta de Maz-Machado et al. (2013) y Madrid et al. (2015).

El tipo de representación más abundante es el lenguaje verbal. Se usan las palabras para definir conceptos, enunciar propiedades y resulta- 
dos, realizar demostraciones, proponer ejemplos, ejercicios y problemas. Las demostraciones de propiedades y resultados se realizan a través de este tipo de representación, aunque apoyadas en expresiones simbólicas y numéricas. Por ejemplo: "Acabamos de ver que por medio del lenguaje simbólico ó razonamiento artificial se simplifica mucho la resolucion de los problemas: este lenguaje simbólico ó razonamiento artificial, aplicado á la resolucion de los problemas matemáticos, se llama Álgebra" (Cortázar, 1865, p. 5).

Las representaciones algebraicas combinan números con signos y letras. Estas se usan frecuentemente para expresar expresiones algebraicas y ecuaciones con el objetivo de clarificar propiedades, realizar demostraciones y dar ejemplos (figura 4).

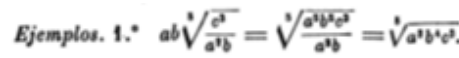

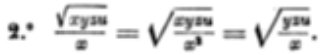

Figura 4. Representación algebraica en el Tratado de Álgebra Elemental de Juan Cortázar, 1865, p. 101.

Para dar ejemplos, mediante números y símbolos, de las diferentes propiedades, se usan representaciones numéricas. Aparecen en muy pocas ocasiones debido a que se trata de un Tratado de Álgebra y para enunciar ejemplos es más frecuente el uso de la representación algebraica (figura 5).

$$
\begin{aligned}
& \text { Ejenplos. } 10^{\circ} \quad 14-(-6)=14+6=20 \text {. } \\
& \text { 2. }-14-(-6)=-14+6=-8 \text {. }
\end{aligned}
$$

Figura 5. Representación algebraica en el Tratado de Álgebra Elemental de Juan Cortázar, 1865, p. 8.

Por último, y aunque solo en dos ocasiones, se observan gráficas para ayudar a resolver problemas de móviles en la que las letras indican la posición de cada uno de los móviles (figura 6).

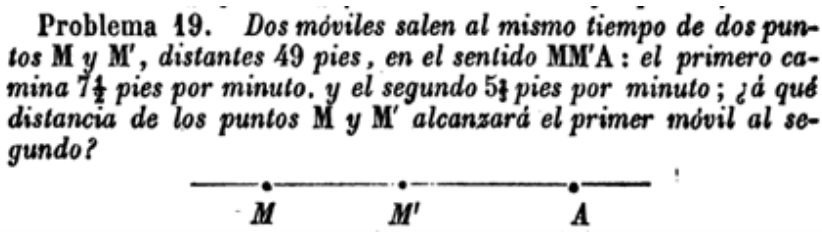

Figura 6. Representación gráfica en el Tratado de Álgebra Elemental de Juan Cortázar, 1856, p. 5. 


\section{e. Análisis fenomenológico}

Se han encontrado nueve tipos de fenómenos o contextos a través de los cuales Cortázar ejemplifica los conceptos que presenta fenómenos de: desplazamientos, capacidad, cronológicos, comerciales, laborales, transmisión, financieros, lúdicos y estrictamente matemáticos, tanto aritméticos como algebraicos. Esta clasificación coincide parcialmente con la realizada por Madrid y Maz-Machado (2015).

La obra se desarrolla en un contexto fundamentalmente matemático a la hora de dar explicaciones de las operaciones, realizar demostraciones, enunciar ejemplos y proponer ejercicios y problemas. Se manifiestan de dos formas distintas:

- Aritméticos: para mostrar las operaciones de adición, sustracción, multiplicación y división de los números negativos, raíces y potencias de números positivos y negativos, resolver problemas de tipo aritmético o calcular valores numéricos de expresiones algebraicas.

- Algebraicos: para resolver las operaciones con expresiones algebraicas, resolver ecuaciones, potencias y raíces de las cantidades algebraicas o expresar los términos generales de las progresiones.

Como complemento a los fenómenos matemáticos, encontramos contextos en los que hay que resolver problemas de situaciones que se encuentran en la naturaleza y se rigen mediante leyes físicas. Estos son de tres tipos:

- Desplazamientos: para calcular distancias recorridas en ambos sentidos de la recta o la circunferencia (avances o retrocesos) bajo unas condiciones dadas. Por ejemplo, "Problema $9^{\circ}$.Siendo en un reloj las 12 en punto, y estando por consiguiente el minutero sobre el horario, ¿qué hora será cuando el minutero vuelva á colocarse sobre el horario?" (Cortázar, 1865, p. 77).

- Capacidad: para calcular los tiempos de llenado o vaciado de productos o sustancias en lugares o recipientes.

Problema $8^{\circ}$.Se ha llenado de agua en 12 minutos una vasija de 39 azumbres de cabida, habiéndola espuesto primeramente á un caño que arrojaba 3 azumbres de agua en cada minuto, y despues á otro que arrojaba 4 azumbres de agua en cada minu- 
El Tratado de Álgebra elemental de Juan Cortázar: un libro significativo para la enseñanza de las matemáticas en España

to. ¿Cuántos minutos estuvo expuesta á cada uno de los caños? (Cortázar, 1865, p. 76).

- Cronológicos: para calcular la edad o edades de personajes y, para ello se aportan las relaciones entre las edades de dos o varios de ellos o las condiciones entre diferentes periodos de su vida. Por ejemplo, "Problema 50. Preguntándole á uno qué edad tenia un hijo suyo, respondió: si del doble de su edad se resta el triplo de que tenía 6 años há, resultará su edad actual ¿Cuántos años tenia el hijo?" (Cortázar, 1865, p. 75).

Aquellas situaciones que implican la distribución de bienes y servicio, las denominamos situaciones económicas. Encontramos cuatro tipos:

- Fenómenos comerciales: aquellos que se refieren a situaciones en las que hay implicadas operaciones de compra-venta. Por ejemplo, "Problema 18. ¿Cuántas fanegas de trigo de á 50 reales y de á 40 reales se han de mezclar, para tener trigo de á 47 reales, escediendo el primer número al segundo en 30 fanegas?" (Cortázar, 1865, p. 83).

- Fenómenos laborales: aquellos que se refieren a situaciones en las que se debe calcular el salario a percibir por una o varias personas.

2. ${ }^{\circ}$ Un particular ha pagado 60 reales por 6 días de trabajo de un obrero y 5 días de trabajo de otro. En otra ocasion, ganando los obreros el mismo jornal, les ha pagado 184 reales por 17 días de trabajo del primero trece del segundo. Se pregunta, cuál fué el jornal de estos obreros. (Cortázar, 1865, p. 92).

- Fenómenos de transmisión: aquellos en los que debemos averiguar las partes correspondientes al reparto de una herencia.

Problema 11. Dispuso uno en su testamento que del capital que dejaba, se diesen al mayor de sus hijos 1000 duros y la décima parte del resto; que al hijo segundo se diesen 2000 duros y la décima parte del resto; al tercero 3000 duros y la décima parte del resto, y así sucesivamente. Hecho el reparto, se vió que todas las partes eran iguales. Se pregunta, ¿cuánta era toda la herencia, cuántos eran los hijos y cuánto correspondió á cada uno? (Cortázar, 1865, p. 78). 
- Fenómenos financieros: aquellos en los que se presentan situaciones en las que se debe determinar una ganancia o una pérdida económica. Por ejemplo, "255. Hallar la relacion que liga al capital, al tanto por 1 anual, al tiempo y á la suma del capital é intereses al cabo de este tiempo." (Cortázar, 1865, p. 209).

Igualmente encontramos fenómenos que implican el cálculo del ganador de un juego o las ganancias obtenidas.

Problema 4. ${ }^{\circ}$ Se pusieron dos á jugar con otros, y ambos perdieron, el uno 12 rs. Y el otro 57 rs.: el dinero con que este segundo se levantó del juego era la cuarta parte del que al primero le habia quedado; siendo así que los dos se pusieron á jugar con igual cantidad de dinero. Se pregunta ¿cuál era esta cantidad? (Cortázar, 1865, p. 75).

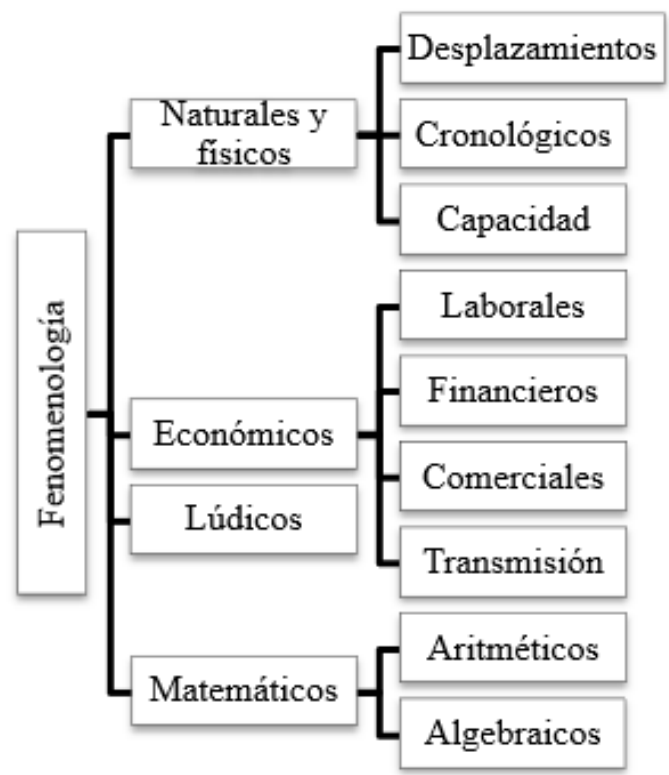

Figura 7. Tipos de fenómenos o situaciones 
El Tratado de Álgebra elemental de Juan Cortázar: un libro significativo para la enseñanza de las matemáticas en España

Carmen LeÓn-Mantero, Alexander Maz-Machado y María José Madrid Martín

\section{Conclusiones}

Podemos considerar al Tratado de Álgebra elemental como una de las obras de mayor importancia para la formación matemática de varias generaciones de españoles durante la segunda mitad del siglo XIX y la primera del siglo XX. Entre los indicadores que muestran la calidad e influencia de la obra, se encuentran las numerosas reediciones, que, entre todos sus libros de texto llegaron al medio millón de ejemplares vendidos, tanto de las publicaciones oficiales impresas en Madrid, como las que fueron impresas sin su consentimiento en ciudades tan importantes como París o Nueva York. La obra contó con el consentimiento y aprobación del gobierno, que la incluyó en la corta lista de libros de texto oficial para uso en Universidades, Institutos y Escuelas Profesionales.

De entre las valoraciones dadas por otros autores sobre las obras, destacan la actualidad de los contenidos con respecto a autores españoles contemporáneos, su buen nivel matemático tanto para los alumnos de la segunda enseñanza como universitaria y sus evidentes influencias de los planes de estudios franceses, debidas a sus años de estudio de ingeniería en París (Vea, 1995).

Cortázar introduce diferencias significativas con respecto a obras contemporáneas. Una de estas consiste en la segregación del Álgebra y la Aritmética, su coherencia al ordenar los contenidos y al señalar con asteriscos los enunciados que los profesores de secundaria debían omitir en sus clases. Esto hace del Tratado de Álgebra elemental una obra versátil en la mayoría de los ámbitos educativos, ya sea en enseñanza secundaria, en escuelas profesionales, en escuelas normales o en la Universidad.

Los contenidos destacan por su carácter y estructura formal. Todos los resultados vienen acompañados de su rigurosa demostración o de la ubicación, en alguna de sus obras, en las que puede hallarse. También por la gran variedad de ejemplos y situaciones que se encuentran, desde el estudio de los números negativos, hasta la resolución de ecuaciones, pasando por el cálculo de expresiones algebraicas.

Al tratarse de una obra sobre Álgebra, el contexto al que más se recurre a la hora de proponer ejemplos y problemas, es el estrictamente matemático. Sin embargo, también podemos encontrar situaciones de tipo económico, físico o natural y lúdico. Desde el punto de vista fenomenológico, presenta una gran variedad de contextos en los que pueden ser aplicados el cálculo de expresiones algebraicas y la resolución de ecuaciones. 
El análisis de contenido, centrado en los sistemas de representación de la obra nos muestra que predomina el lenguaje verbal elegido meticulosamente para exponer definiciones, resultados, propiedades, ejemplos, ejercicios y problemas y el lenguaje simbólico para ejemplificar dichos resultados y ejemplos. En menor medida se hallan representaciones numéricas y gráficas, y es nulo, el uso de tablas y esquemas.

Irueste (1912) y Vea (1995) realizan sendos análisis de la obra y ambos coinciden en que su contenido se ajusta a las pautas comunes de la mayoría de los textos de la época. Estos destacan la originalidad de algunos de sus capítulos, como el cociente de un número entre cero, la indeterminación cero entre cero, el tratamiento de la radicación de monomios y polinomios, la resolución de ecuaciones de segundo grado o los conceptos de máximo y mínimo. El tratamiento dado a las progresiones y los logaritmos coincide con el que se le da en la actualidad y, es que Cortázar considera que los logaritmos deben ser incluidos dentro del álgebra y no dentro de la aritmética como lo hacen Bourdon o Fernández Vallín.

El análisis comparativo realizado por Peset et al. (1978) entre el Manuel des aspirants y los Elementos de Álgebra de M. Bourdon, ambos, libros de texto franceses contemporáneos a este tratado, nos revela que el nivel científico del autor español es inferior que el de los franceses, si bien ha de tenerse en cuenta el contexto histórico en el que nuestro autor redacta su obra. Además, consideran que el texto matemático está escrito de forma esquemática, al contrario de lo que sucede en los textos franceses de la época, que califican como deductivos.

Estudios como el que hemos presentado permiten comprender la forma en la que se transmitían los conocimientos matemáticos en una época pasada y en un contexto geográfico y social determinado, como es el español. También revela la preocupación que existía, ya entonces, por aspectos didácticos que favorecieran el aprendizaje, algo que hoy en día, sigue siendo primordial, tanto en las aulas como en los manuales actuales. 
El Tratado de Álgebra elemental de Juan Cortázar: un libro significativo para la enseñanza de las matemáticas en España

Carmen León-Mantero, Alexander Maz-Machado y María José Madrid Martín

\section{Referencias}

Castro, E. y Castro, E. (1997). Representaciones y modelización. En Rico, L. (coord.), La educación matemática en la enseñanza secundaria (pp. 95-124). Barcelona: Horsori.

Cortázar, J. (1847). Démonstration des analogies de Néper. Nouvelles annales de mathématiques, 6, 218-220. Recuperado el 15 de marzo de 2016 de http://www.numdam. org/item?id=NAM_1847_1_6_218_1

Fauvel, J. (1991). Using History in Mathematics Education. For the Learning of Mathematics, 11(2), 3-6.

Frejd, P. (2013). Old algebra textbooks: a resource for modern teaching. BSHM Bulletin: Journal of the British Society for the History of Mathematics, 28(1), 25-36.

Furinghetti, F. (2000). The history of mathematics as a coupling link between secondary and university teaching. International Journal of Mathematical Education in Science and Technology, 31, 43-51.

Gaceta de Madrid (1848). Madrid. Imprenta Real.

Girón, R. M., y Girón, F. M. (2010). El matemático José Andrés Irueste (1844-1920) y su entorno. La Gaceta de la RSME, 13(2), 353-378.

Gómez, P. (2002). Análisis didáctico y diseño curricular en matemáticas. Revista EMA, $7(3), 251-292$.

Gómez, B. (2011a). Marco preliminar para contextualizar la investigación en historia y educación matemática. Epsilon: Revista De Educación Matemática, 28(1) (77), 9-22.

Gómez, B. (2011b). El análisis de manuales y la identificación de problemas de investigación en Didáctica de las Matemáticas. PNA, 5(2), 49-65.

Irueste, J. A. (1912). D. Juan Cortázar. Revista de la Sociedad Matemática Española, 1(8), 285-290.

Jankvist, U. T. (2009). A categorization of the "whys" and "hows" of using history in mathematics education. Educational studies in Mathematics, 71(3), 235-261.

León-Mantero, C. y Maz-Machado, A. (2015). Juan Cortázar y sus aportaciones a la Educación Matemática española del siglo XIX. Ensayos, Revista de la Facultad de Educación de Albacete, 30(1), 55-62.

Madrid, M.J. (2016). Los libros de aritmética en España a lo largo el siglo XVI, Universidad de Salamanca, Salamanca.

Madrid. M. J. y Maz-Machado, A. (2015). Analysis of two Spanish arithmetic books written in the XVI-century, Journal of Education, Psychology and Social Sciences 3, 117121.

Madrid, M. J., Maz-Machado, A. y León-Mantero, C. (2015). Representations in the Sixteenth-Century arithmetics books, Universal Journal of Education Research 3, 396401.

Maz, A. (2005). Los números negativos en España en los siglos XVIII y XIX. Granada: editorial de la Universidad de Granada.

Maz, A. y Rico, L. (2007). Situaciones asociadas a los números negativos en textos de matemáticas españoles de los siglos XVIII y XIX. PNA, 1(3), 113-123

Maz, A., y Rico, L. (2009a).Números negativos en los siglos XVIII: fenomenología y representaciones. Revista de Investigación Psicoeducativa, 17(1), 117-129. 
El Tratado de Álgebra elemental de Juan Cortázar: un libro significativo para la enseñanza de las matemáticas en España

Carmen León-Mantero, Alexander Maz-Machado y María José Madrid Martín

Maz, A. y Rico, L. (2009b). Las Liciones de Thomas Cerda: doscientos cincuenta años (1758-2008). Suma (60), 35-41.

Maz-Machado, A., y Rico, L. (2013). El Tratado elemental de matemáticas de José Mariano Vallejo en el bicentenario de su publicación. Suma (74), 55-63.

Maz-Machado, A., y Rico, L. (2015). Principios didácticos en textos españoles de matemáticas en los siglos XVIII y XIX. Relime, Revista latinoamericana de Investigación Educativa 18 (1), 49-76.

Peset, J. L., Garma, S., \& Pérez-Garzón, J. S. (1978). Ciencias y enseñanza en la revolución burguesa. Madrid: Siglo veintiuno.

Rico, L. (1997). Los organizadores del currículo de matemáticas. En L. Rico (Coord.), La Educación Matemática en la enseñanza secundaria (pp.39-59). Barcelona: ICEHorsori.

Rico, L., Marín, A., Lupiáñez, J. L., \& Gómez, P. (2008). Planificación de las matemáticas escolares en secundaria. El caso de los números naturales. Suma, 58, 7-23.

Rodríguez, C. (2009). El instituto del Cardenal Cisneros de Madrid (1845-1877).Madrid: CSIC.

Schubring, G, (1987). On the methodolgy of analysing historical textbooks: Lacroix as textbook authors. For the learning of mathematics, 7(3), 41-51.

Schubring, G. (1988). Discussions épistémologiques sur le statut des nombres négatifs et leur représentation das les manuels allemands et français de mathématique entre 1795 et 1845 . Actes du premier colloque franco-allemand de didáctique des mathématiques et de l'informatique (pp. 137-145). Editions La Pensée Sauvage.

Sierra, M., González, M.T. y López (1999). Evolución histórica del concepto de límite funcional en los libros de teto de Bachillerato y Curso de Orientación Universitaria (COU):1940-1995. Enseñanza de las ciencias, 17(3), 463-476.

Sierra, M., González, M.T. y López, C. (2003). El concepto de continuidad en los manuales españoles de enseñanza secundaria de la segunda mitad del siglo XX. Educación Matemática, 15(1), 21-50.

Tzanakis, C. y Arcavi, A. (2000). Integrating history of mathematics in the classroom: an analytic survey. En J. Fauvel y J. van Maanen (Eds). History in Mathematics Education The ICMI Study (p. 201-240). Dordrecht: Kluwer Academic Publishers.

Universidad de Madrid (1856). Anuario de la Universidad Central para el curso 18561857. Madrid: Autor.

Universidad de Madrid (1857). Anuario de la Universidad Central para el curso 18571858. Madrid: Autor.

Vea, F. (1995). Las matemáticas en la enseñanza secundaria en España (s. XIX). Zaragoza: Universidad de Zaragoza.

Vicuña, G. (1875). Cultivo actual de las ciencias físico-matemáticas en España. Discurso leído en la Universidad Central en el acto de apertura del curso académico 18751876. Madrid: Imprenta de José M. Ducazcal.

Wussing, H. (1998). Lecciones de historia de las matemáticas.Madrid: Siglo XXI. 
